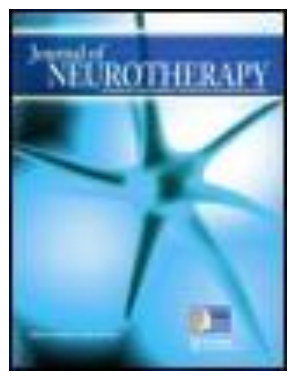

Journal of Neurotherapy: Investigations in Neuromodulation, Neurofeedback and Applied Neuroscience

\title{
Low Resolution Brain Electromagnetic Tomography (LORETA)
}

Roberto D. Pascual-Marqui PhD ${ }^{\mathrm{a}}$

${ }^{a}$ KEY Institute for Brain-Mind Research, University Hospital of Psychiatry, Zurich, Switzerland

Published online: 20 Oct 2008.

To cite this article: Roberto D. Pascual-Marqui PhD (2001) Low Resolution Brain Electromagnetic Tomography (LORETA), Journal of Neurotherapy: Investigations in Neuromodulation, Neurofeedback and Applied Neuroscience, 4:4, 31-33, DOI: 10.1300/J184v04n04_05

To link to this article: http://dx.doi.org/10.1300/J184v04n04_05

\section{PLEASE SCROLL DOWN FOR ARTICLE}

(c) International Society for Neurofeedback and Research (ISNR), all rights reserved. This article (the "Article") may be accessed online from ISNR at no charge. The Article may be viewed online, stored in electronic or physical form, or archived for research, teaching, and private study purposes. The Article may be archived in public libraries or university libraries at the direction of said public library or university library. Any other reproduction of the Article for redistribution, sale, resale, loan, sublicensing, systematic supply, or other distribution, including both physical and electronic reproduction for such purposes, is expressly forbidden. Preparing or reproducing derivative works of this article is expressly forbidden. ISNR makes no representation or warranty as to the accuracy or completeness of any content in the Article. From 1995 to 2013 the Journal of Neurotherapy was the official publication of ISNR (www. Isnr.org); on April 27, 2016 ISNR acquired the journal from Taylor \& Francis Group, LLC. In 2014, ISNR established its official open-access journal NeuroRegulation (ISSN: 2373-0587; www. neuroregulation.org).

THIS OPEN-ACCESS CONTENT MADE POSSIBLE BY THESE GENEROUS SPONSORS

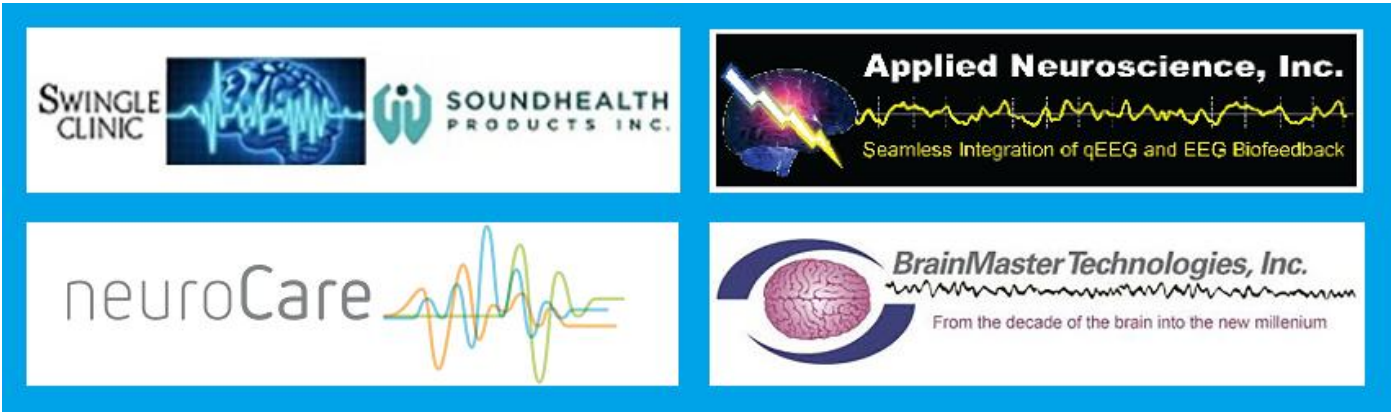




\section{TECHNICAL NOTES}

\section{Low Resolution Brain Electromagnetic Tomography (LORETA): The Technique, Its Validation, and Methods of Analysis}

Roberto D. Pascual-Marqui, PhD

Multi-channel recordings of scalp electric potential differences (EEG/ ERPs) provide insufficient information for determining uniquely the three-dimensional distribution of electric neuronal activity in the brain. This means that infinitely many possible solutions exist for this inverse problem. Many particular solutions have been published in the literature, each one characterized by its own set of assumptions.

It is a well-known fact from electrophysiology that the fundamental reason why EEG can be measured on the scalp is the occurrence of highly synchronized post-synaptic potentials in clusters of cortical pyramidal neurons. By taking this single electrophysiological fact into account

Roberto D. Pascual-Marqui is affiliated with The KEY Institute for Brain-Mind Research, University Hospital of Psychiatry, Zurich, Switzerland.

This material is an abstract of scientific research presented by Dr. Pascual-Marqui at the SNR 2000 Conference in St. Paul, MN.

For a complete discussion of LORETA and other examples, plus literature references and reprints see: http://www.unizh.ch/keyinst/NewLORETA/LORETA01.htm\# WhatIsLORETA 
(i.e., synchronization) a new inverse solution is obtained: low-resolution brain electromagnetic tomography (LORETA).

With the development of LORETA, it can now be shown that the scalp electric potential provides sufficient information for obtaining a low spatial resolution estimate of the electric neuronal activity. From a mathematical viewpoint, LORETA yields a spatial low-pass filtered version of the cortical current density, with correct localization. From an electrophysiological viewpoint, LORETA is consistent with the highly synchronized activity of neighboring neuronal populations. From an empirical viewpoint, functional LORETA images yield correct neuroanatomical localization in visual and auditory evoked potential experiments.

The LORETA method currently implemented makes use of the human head model described in the Talairach brain atlas. By means of cross-registration techniques, EEG measurements made on any human head can be adjusted to the geometry of the Talairach reference brain.

A LORETA image corresponding to the P100 visual evoked potential peak (data kindly provided by Koenig and Lehmann, 1996) is shown in Figure 1 (maximum current density in Brodmann area 17).

Once the validity of the method is established, LORETA can be used as a relatively trustworthy tool for the study of brain function, as is carried out with other well-established methods, such as PET and fMRI. However, unlike PET and fMRI, which provide metabolic information, LORETA images provide high time resolution electric neuronal activity information.

Methods of analysis of LORETA images for event related potential (ERP) data and spontaneous EEG data are reviewed. In the case of ERP data, LORETA images are usually computed at peak latencies, offering information about the location and distribution of the electrically active neurons that generated the ERP component. Instantaneous LORETA images can also be computed for EEG. These images are of interest when analyzing epileptic spikes, for example. However, it is also often relevant to compute "frequency band" LORETA images, which offer information about the location and distribution of the electrically active neurons that generated the alpha rhythm, for example. 


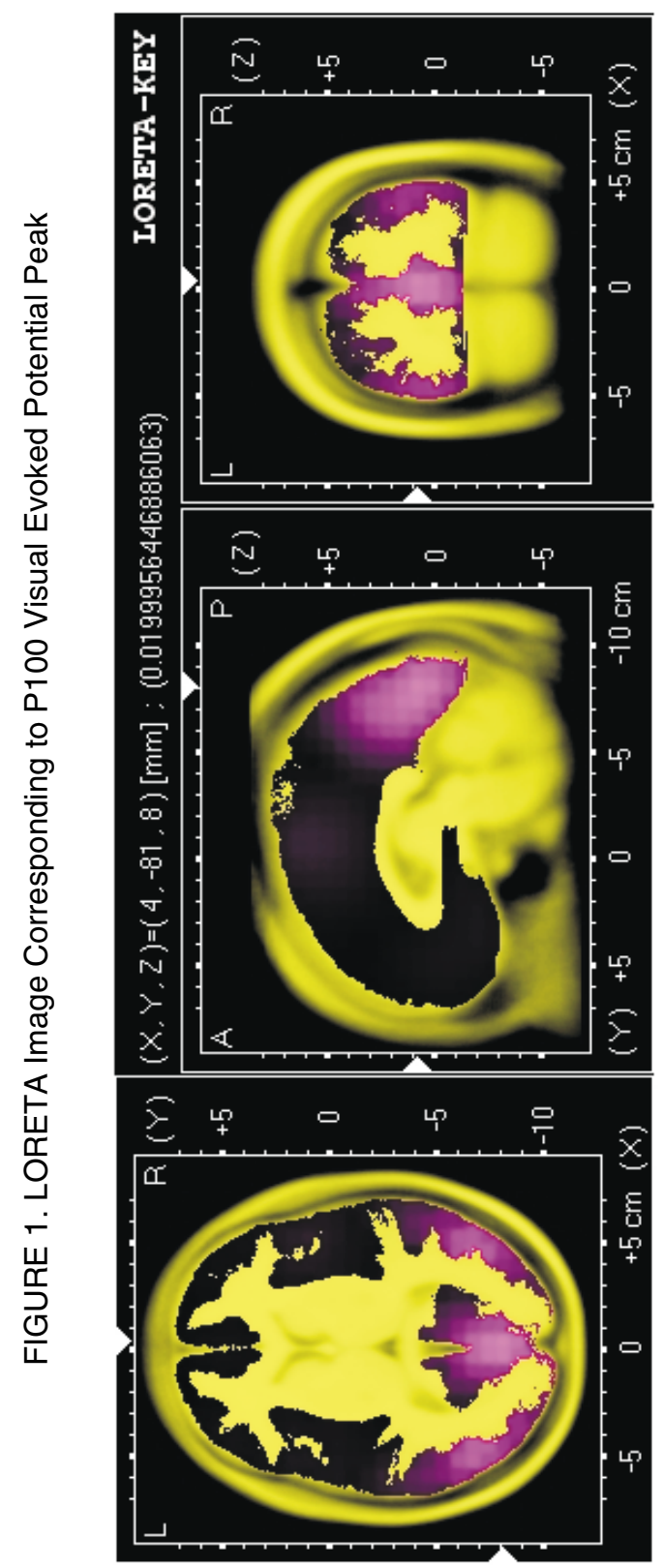

